\title{
MEASURING THE REDSHIFT DEPENDENCE OF THE COSMIC MICROWAVE BACKGROUND MONOPOLE TEMPERATURE WITH PLANCK DATA
}

\author{
I. De Martino ${ }^{1}$, F. Atrio-Barandela ${ }^{1}$, A. Da Silva ${ }^{2}$, H. Ebeling ${ }^{3}$, A. Kashlinsky ${ }^{4}$, D. Kocevski ${ }^{5}$, And C. J. A. P. Martins ${ }^{2}$ \\ ${ }^{1}$ Física Teórica, Universidad de Salamanca, E-37008 Salamanca, Spain; ivan.demartino@usal.es, atrio@usal.es \\ ${ }^{2}$ Centro de Astrofisica da Universidade do Porto, Rua das Estrelas s/n, 4150-762 Porto, Portugal; asilva @ astro.up.pt, Carlos.Martins@astro.up.pt \\ ${ }^{3}$ Institute for Astronomy, University of Hawaii, 2680 Woodlawn Drive, Honolulu, HI 96822, USA; ebeling @ ifa.hawaii.edu \\ ${ }^{4}$ SSAI and Observational Cosmology Laboratory, Code 665, Goddard Space Flight Center, Greenbelt, MD 20771, USA; alexander.kashlinsky@nasa.gov \\ ${ }^{5}$ Department of Physics, University of California at Davis, 1 Shields Avenue, Davis, CA 95616, USA; kocevski@ physics.ucdavis.edu \\ Received 2012 February 24; accepted 2012 July 18; published 2012 September 12
}

\begin{abstract}
We study the capability of Planck data to constrain deviations of the cosmic microwave background (CMB) blackbody temperature from adiabatic evolution using the thermal Sunyaev-Zeldovich anisotropy induced by clusters of galaxies. We consider two types of data sets depending on how the cosmological signal is removed: using a CMB template or using the $217 \mathrm{GHz}$ map. We apply two different statistical estimators, based on the ratio of temperature anisotropies at two different frequencies and on a fit to the spectral variation of the cluster signal with frequency. The ratio method is biased if CMB residuals with amplitude $\sim 1 \mu \mathrm{K}$ or larger are present in the data, while residuals are not so critical for the fit method. To test for systematics, we construct a template from clusters drawn from a hydro-simulation included in the pre-launch Planck Sky Model. We demonstrate that, using a proprietary catalog of X-ray-selected clusters with measured redshifts, electron densities, and X-ray temperatures, we can constrain deviations of adiabatic evolution, measured by the parameter $\alpha$ in the redshift scaling $T(z)=T_{0}(1+z)^{1-\alpha}$, with an accuracy of $\sigma_{\alpha}=0.011$ in the most optimal case and with $\sigma_{\alpha}=0.018$ for a less optimal case. These results represent a factor of 2-3 improvement over similar measurements carried out using quasar spectral lines and a factor 6-20 with respect to earlier results using smaller cluster samples.
\end{abstract}

Key words: cosmic background radiation - cosmology: observations - cosmology: theory

\section{INTRODUCTION}

One of the fundamental tenets of the big bang paradigm is the adiabatic evolution of the universe. Early thermal equilibrium among the different particle species, entropy, and photon number conservation produces a cosmic microwave background (CMB) with a blackbody spectrum. The CMB temperature was measured to be $T_{0}=2.725 \pm 0.002 \mathrm{~K}$ by the Far Infrared $\mathrm{Ab}$ solute Spectrometer (FIRAS) of the Cosmic Background Explorer satellite (Mather et al. 1999). The adiabatic expansion of the universe and photon number conservation imply that the CMB temperature evolves with redshift as $T(z)=T_{0}(1+z)$. Establishing observationally this relation would test our current understanding of the universe since models like decaying vacuum energy density and gravitational "adiabatic" photon creation predict different scaling relations (Overduin \& Cooperstock 1998; Matyjasek 1995; Lima et al. 2000; Puy 2004; Jetzer et al. 2011; Jetzer \& Tortora 2012). In these models, energy is slowly injected without producing distortions on the blackbody spectrum, evading the tight FIRAS constraints. Nevertheless, in these models the blackbody temperature scales nonlinearly as $T(z)=T_{0}(1+z)^{1-\alpha}$. Therefore, measuring the redshift dependence of the CMB blackbody temperature at various cosmological epochs can provide strong constraints on physical theories at the fundamental level.

There are currently two methods to determine $T(z)$ at redshifts $z>0$ : (1) using fine-structure lines from interstellar atoms or molecules, present in quasar spectra, whose transition energies are excited by the CMB photon bath (Bahcall \& Wolf 1968) and (2) from the thermal Sunyaev-Zeldovich anisotropies (hereafter TSZ; Sunyaev \& Zeldovich 1972, 1980) due to the inverse Compton scattering of photons by the free electrons within the potential wells of clusters of galaxies. Early observations of fine-structure levels of atomic species like carbon only led to upper limits on $T(z)$ because the CMB is not the only radiation field populating the energy levels and collisional excitation is an important contribution. Assuming that the CMB is the only source of excitation, Songaila et al. (1994) measured $T(z=1.776)=7.4 \pm 0.8 \mathrm{~K}$; but collisional excitation was not negligible and it had to be corrected. The first unambiguous measurement was only achieved six years later, with a considerably larger error bar (Srianand et al. 2000). Lately, Noterdaeme et al. (2011) succeeded in obtaining direct and precise measurements from the rotational excitation of $\mathrm{CO}$ molecules. They constrained the deviation from linear scaling to be $\alpha=-0.007 \pm 0.027$ at $z \sim 3(\alpha=0$ corresponds to the standard redshift scaling). Battistelli et al. (2002) reported the first observations of $T(z)$ using the TSZ effect of the COMA and A2163 clusters of galaxies with $\alpha=-0.16_{-0.32}^{+0.34}$. Luzzi et al. (2009) determined the CMB temperature in the redshift range $z=0.023-0.546$, from the measurements of 13 clusters. They restricted their analysis to $\alpha \in[0,1]$ and set up an upper limit of $\alpha \leqslant 0.079$ at the $68 \%$ confidence level. No significant deviations from the redshift dependence of the CMB temperature predicted in the standard model have been found.

While there is interest in doing such observations as far back as possible (which one can do with spectroscopic methods), low-redshift measurements also play an important role. First, the two techniques are complementary with each other since they have different systematics and probe the adiabatic evolution of the universe at different redshifts. Spectroscopic observations probe the matter era, roughly between redshifts $z=2-4$, while TSZ probes the epoch of dark energy domination, $z \leqslant 1$. In particular, these measurements can shed light on the onset of dark energy domination; in many models this is associated with a phase transition (Mortonson et al. 2009; Nunes et al. 2009) 
which could leave imprints in the $T(z)$ relation. Second, in models where photon number is not conserved, not only does the temperature-redshift relation change, but so does the distance duality relation (Etherington 1933), and these departures from the standard behavior are not independent. This link between the two relations requires information at all redshifts and will, when better data sets become available, be a powerful consistency test for the standard cosmological paradigm (Avgoustidis et al. 2012).

The Planck mission has been designed to produce a full-sky survey of the CMB with unprecedented accuracy in temperature and polarization (Planck Collaboration 2011a). The instrument operates at nine frequencies logarithmically spaced in the range 30-857 GHz. The in-flight performance of the High and Low Frequency Instruments (HFI and LFI) has been described by the Planck HFI Core Team (2011a) and Mennella et al. (2011). Due to its large frequency coverage, high resolution, and low noise, it is a powerful instrument for blind detection of clusters using the TSZ effect. The first clusters detected by Planck include 189 cluster candidates with signal-to-noise ratio $(\mathrm{S} / \mathrm{N})$ larger than 6 (Planck Collaboration 2011b). These SZ clusters are mostly at moderate redshifts $(86 \%$ had $z<0.3)$ and span over a decade in mass, up to the rarest and most massive clusters with masses above $10^{15} M_{\odot}$. In this article, we analyze how Planck data can be used to test the standard scaling relation of the CMB temperature with redshift. We use a two-fold approach: first, our pipeline is tested on simulated clusters drawn from a full hydrodynamical simulation; second, using a catalog of 623 clusters derived from ROSAT data and with well measured X-ray properties, we predict with what precision $\alpha$ will be measured by Planck using those clusters. In comparison with earlier analysis of Horellou et al. (2005), we use a catalog of X-ray-selected clusters, and in our simulations gas evolution is fully taken into account. Briefly, in Section 2 we describe our methodology; in Section 3 we discuss the construction of our simulated data; in Section 4 we explain our pipeline; in Section 5 we present our results; and in Section 6 we summarize our main conclusions.

\section{METHOD}

Compton scattering of CMB photons by the hot intracluster (IC) gas induces secondary temperature anisotropies on the $\mathrm{CMB}$ radiation in the direction of clusters of galaxies. There are two components: the thermal (TSZ, Sunyaev \& Zeldovich 1972) due to the thermal motion of the IC medium with temperature $T_{e}$ and the kinematic (KSZ, Sunyaev \& Zeldovich 1980) due to the motion of the cluster with speed $\boldsymbol{v}_{c l}$ respect to the isotropic CMB frame. Neglecting higher order corrections, the TSZ and KSZ contributions to the temperature anisotropy in the direction of a cluster, given by the unit vector $\mathbf{n}$, are

$$
\frac{T(\hat{n})-T_{0}}{T_{0}}=\int\left[G(v) \frac{k_{B} T_{e}}{m_{e} c^{2}}-\frac{\boldsymbol{v}_{c l} \hat{n}}{c}\right] d \tau=G(v) y_{c}-\tau \frac{\boldsymbol{v}_{c l} \hat{n}}{c} .
$$

In this expression, $d \tau=\sigma_{T} n_{e} d l$ is the cluster optical depth, $n_{e}(l)$ is the electron density evaluated along the line of sight $l, \sigma_{T}$ is Thomson cross section, $T_{0}$ is the current CMB mean temperature, $k_{B}$ is the Boltzmann constant, $m_{e} c^{2}$ is the electron annihilation temperature, $c$ is the speed of light, and $v$ is the frequency of observation. The Comptonization parameter is defined as $y_{c}=\left(k_{B} \sigma_{T} / m_{e} c^{2}\right) \int n_{e} T_{e} d l$. Due to its frequency dependence $G(v)$, the TSZ is a distortion of the CMB spectrum. Its amplitude is independent of the cluster distance, making it a useful tool to detect clusters at high redshifts. All known astrophysical foregrounds have a different dependence with frequency so clusters can be clearly detected in CMB maps with enough frequency coverage. In the non-relativistic limit, $G(x)=x \operatorname{coth}(x / 2)-4$, but for very massive clusters, relativistic corrections need to be included. The reduced frequency $x$ is given by $x=h v(z) / k T(z)$ with $v(z)$ the frequency of a CMB photon scattered off by the IC gas and $T(z)$ the blackbody temperature of the CMB at the cluster location.

If the universe evolves adiabatically, $T(z)=T_{0}(1+z)$; due to the expansion, the frequency of a photon scattered by the IC plasma at redshift $z$ is Doppler shifted as $v(z)=v_{0}(1+z)$ and the ratio $x=h v(z) / k T(z)=h v_{0} / k T_{0}=x_{0}$ is independent of redshift. If the evolution of the universe is non-adiabatic, the temperature-redshift relation would not be constant. Two functional forms have been considered in the literature: $T(z)=$ $T_{0}(1+z)^{1-\alpha}$ (Lima et al. 2000) and $T(z)=T_{0}(1+b z)$ (LoSecco et al. 2001). In both cases, the photon frequency is assumed to be redshifted as in the standard model: $v(z)=v_{0}(1+z)$. Since the largest fraction of known clusters of galaxies are at redshifts below $z \leqslant 0.7-1$, the differences between both redshift dependences are small so we will only analyze the first model. The reduced frequency varies as $x=x_{0}(1+z)^{\alpha}$ and the spectral frequency dependence of the TSZ effect, $G(v)$ now depends on $\alpha: G(x)=G(v, \alpha)(\alpha=0$ corresponds to adiabatic evolution). Fabbri et al. (1978) proposed to measure the zero cross frequency of clusters at different redshifts that, for adiabatic evolution, occurs at $v \simeq 217 \mathrm{GHz}$. Rephaeli (1980) suggested the use of the ratio of the TSZ anisotropy at different scales, $R\left(v_{1}, v_{2}, \alpha\right)=G\left(v_{1}, \alpha\right) / G\left(v_{2}, \alpha\right)$. Both methods have different systematics. By taking ratios, the dependence on the Comptonization parameter is removed and the need to account for model uncertainties on the gas density and temperature profile is avoided. At the same time, the analysis is more complicated since the distribution of temperature anisotropy ratios is highly non-Gaussian (Luzzi et al. 2009). The measurement of the cross over frequency is also problematic since the TSZ is inherently weak and could be dominated by uncertain systematics. For this reason, the measurements carried out thus far (Battistelli et al. 2002; Luzzi et al. 2009), based on a small number of clusters, have concentrated in the ratio method. Zemcov et al. (2012) showed the intrinsic difficulties of measuring the TSZ null frequency in clusters. As an alternative to the zero frequency method, we will fit the TSZ signal at different frequencies and we will measure the function $G(v, \alpha)$. We shall denote this procedure the fit method.

The function $G(v, \alpha)$ characterizes uniquely the TSZ contribution. At each frequency, Planck LFI receivers and HFI bolometers are sensitive to a wide range of frequencies and the spectral dependence is not $G(v)$ but

$$
\bar{G}\left(v_{0}, \alpha\right)=\int_{0}^{\infty} G(\nu, \alpha) e^{\left(\nu-v_{0}\right)^{2} / 2 \sigma_{v_{0}}^{2}} d v .
$$

Hereafter, we will remove the upper bar and $G(v, \alpha)$ will refer to the averaged frequency dependence of Equation (2). In Figure 1 we plot the temperature ratio (Figure 1(a)) and frequency dependence (Figure 1(b)) for different values of $\alpha$. In Figure 1(a), the solid line represents the adiabatic evolution model $\alpha=0$ that is independent of redshift; dot-dashed lines bound the region where $\alpha=-1,1$. From top to bottom, the ratios are $R(v, 353 \mathrm{GHz}, \alpha)$ with $v=143,100,44 \mathrm{GHz}$. In Figure 1(b), we plot the spectral dependence $G(v, \alpha)$ for adiabatic evolution ( $\alpha=0$, dashed line) and $\alpha=-1,1$ for a cluster at redshift $z=0.1$ (dot-dashed line) and $z=0.3$ 

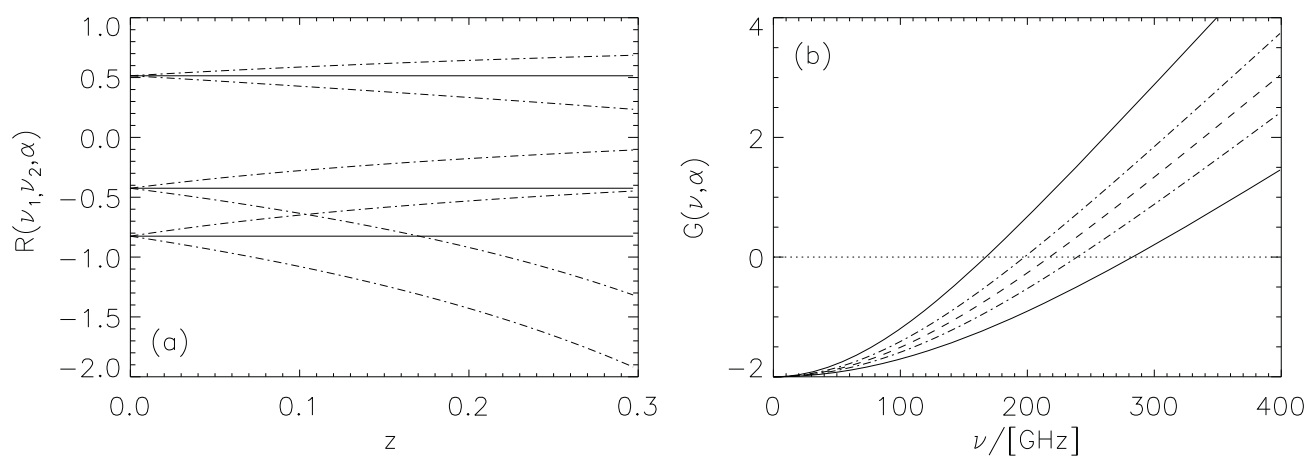

Figure 1. (a) Variation of the ratio $R(v, 353 \mathrm{GHz}, \alpha)$ as a function of redshift for $v=143 \mathrm{GHz}$ (top set of curves), $100 \mathrm{GHz}$ (middle set), and $44 \mathrm{GHz}$ (lower set). The solid straight line corresponds to adiabatic evolution $\alpha=0$ and the dot-dashed lines represent $\alpha=-1,1$. (b) Spectral dependence of the TSZ effect $G(v, \alpha)$ for two clusters located at $z=0.3$ (solid lines) and $z=0.1$ (dot-dashed lines) with $\alpha=-1,1$. The dashed line corresponds to adiabatic evolution $(\alpha=0$ ), identical at all redshifts. The zero cross frequency occurs at the crossing with the dotted line.

Table 1

Technical Details of Planck Channels Used in this Study

\begin{tabular}{lcccccc}
\hline \hline Planck Channel & 1 & 2 & 3 & 4 & 5 & 6 \\
\hline Central frequency $v_{0} / \mathrm{GHz}$ & 44 & 70 & 100 & 143 & 217 & 353 \\
Frequency resolution $\Delta \nu_{0}(\mathrm{FWHM} / \mathrm{GHz})$ & 8.8 & 14 & 33 & 47 & 72 & 116 \\
Angular resolution $\Delta \theta(\mathrm{FWHM} / \mathrm{arcmin})$ & 26.8 & 13.1 & 9.8 & 7.1 & 5.0 & 5.0 \\
Noise per pixel $\sigma_{\text {noise }} / \mu \mathrm{K}$ (Blue Book) & 51 & 52 & 15 & 12 & 19 & 58 \\
Noise per pixel $\sigma_{\text {noise }} / \mu \mathrm{K}$ (in-flight performance) & 109 & 96 & 14 & 9 & 13 & 49 \\
\hline
\end{tabular}

Notes. The noise per pixel in-flight performance corresponds to one year of integration.

(solid line). The null TSZ signal, represented by the dotted line, shows that the zero cross frequency varies in the range $v \sim 170-270 \mathrm{GHz}$.

To construct a pipeline that implements the ratio or zero cross frequency tests we need to consider the specifics of the Planck data. The cosmological CMB signal is the dominant contribution except at the most massive clusters. Foreground residuals or astrophysical contaminants, while smaller in amplitude, would induce systematic shifts in the Comptonization parameter (Aghanim et al. 2005) varying with frequency and biasing the redshift dependence of the TSZ effect. To characterize the noise and foreground emission, the HFI and LFI core teams have constructed maps with the CMB cosmological contribution subtracted off. They have used six different component separation algorithms to construct templates of the CMB cosmological signal to remove this component from the data. The difference between the six methods provides an estimate the CMB residual (Leach et al. 2008; see also Planck HFI Core Team 2011b; Zacchei et al. 2011). The different techniques left residuals with different power spectra and rms in the range 4-16 $\mu \mathrm{K}$, depending on the channel. With respect to astrophysical contaminants, Wilkinson Microwave Anisotropy Probe (WMAP) used the K, Ka Differencing Assemblies, and the extinction corrected $\mathrm{H} \alpha$ maps (Finkbeiner 2003) to subtract the synchrotron and free-free emissions and the Finkbeiner et al. (1999) map to subtract the dust contribution (see Gold et al. 2009 for details). A similar analysis using the 30,545, and $857 \mathrm{GHz}$ channels, and foreground templates could be used to remove the foreground contribution. After the different components have been separated, the final maps will contain residual cosmological signal, residual foreground emission, and noise.

The technical details of the maps considered in this study are listed in Table 1: central frequency $v_{0}$ and FWHM of the antenna spectral response function, approximated by a Gaussian with FWHM $\Delta v_{0}$, angular resolution, and noise per pixel. We indicate the required Blue Book specifications ${ }^{6}$ and the in-flight measured noise per pixel after one year of integration (Mennella et al. 2011; Planck HFI Core Team 2011a). Due to the scanning strategy, the noise is rather inhomogeneous, largely dominated by a white noise component plus a $1 / f$ contribution. In the LFI instrument, the $1 / f$ noise is largest at $30 \mathrm{GHz}$. For the HFI, the noise is largest at 545 and $857 \mathrm{GHz}$. Also, those channels have the smallest resolution and will not be considered in this work. Later we shall show that this is not a limitation since the channels with the highest resolution are the ones with the largest statistical power to determine $\alpha$.

Maps free of the intrinsic CMB signal and foreground residuals are the most convenient to test the adiabatic evolution of the universe, but removing the cosmological CMB signal using statistical algorithms leaves an unknown level of CMB residuals, whose distribution and power spectrum are difficult to model. Alternatively, the intrinsic CMB can be removed exactly by subtracting the highest resolution map, conveniently degraded, from the other maps. Both techniques have different systematics and the consistency of the results would be a test of their validity. Therefore, we shall carry out two types of simulations, depending on what data sets become available: (A) $\mathrm{CMB}$ and foregrounds are removed using templates, to produce maps with only instrumental noise, KSZ, and TSZ with some unknown levels of primordial CMB and foreground residuals. Deviations from adiabatic evolution can be measured by taking ratios of temperature anisotropies at different frequencies (Figure 1(a)) or by fitting the spectral dependence $G(\alpha, v)$ (Figure 1(b)) to the data. (B) The primordial $\mathrm{CMB}$ and KSZ components are removed using the foreground clean $217 \mathrm{GHz}$ map. The angular resolution of the $217 \mathrm{GHz}$ channel is degraded to that of the other five channels before

\footnotetext{
6 http://www.rssd.esa.int/SA/PLANCK/docs/Bluebook-ESASCI(2005)1_V2.pdf
} 

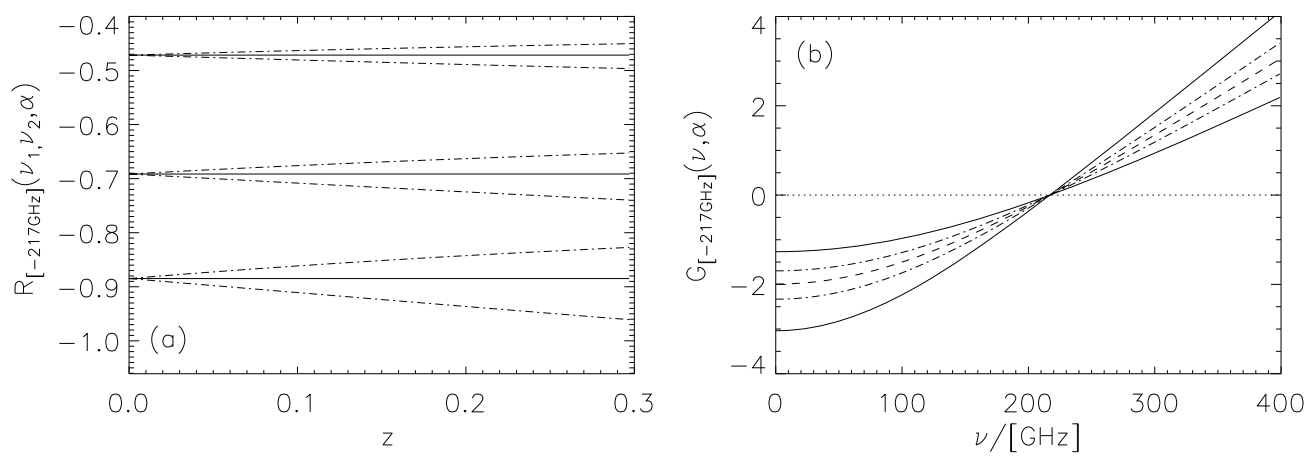

Figure 2. (a) Ratio $R_{[-217 \mathrm{GHz}]}(v, 353 \mathrm{GHz}, \alpha)$ for $v=143,100$, and $44 \mathrm{GHz}$, and (b) spectral dependence of $G(v, \alpha)-G(217 \mathrm{GHz}, \alpha)$. Curves follow the same convention as in Figure 1.

subtracting it from the corresponding map. We checked that the intrinsic $\mathrm{CMB}$ and $\mathrm{KSZ}$ anisotropies are removed exactly but the frequency dependence of the TSZ effect is modified. In Figure 2(a), we plot the ratio of the CMB-KSZ removed maps at different frequencies: $R_{[-217 \mathrm{GHz}]}\left(v_{1}, v_{2}, \alpha\right)=\left[G\left(v_{1}, \alpha\right)-\right.$ $G(217 \mathrm{GHz}, \alpha)] /\left[G\left(v_{2}, \alpha\right)-G(217 \mathrm{GHz}, \alpha)\right]$. In Figure 2(b) we represent $G_{[-217 \mathrm{GHz}]}(\nu, \alpha)=G(\nu, \alpha)-G(217 \mathrm{GHz}, \alpha)$. The lines follow the same conventions as in Figure 1. The two data sets, (A) and (B), will have different systematics and we will analyze both to check for consistency.

\section{CLUSTER TEMPLATES AND FINAL MAPS}

We construct two cluster templates: (1) with a sample of real clusters we will predict the statistical power of Planck to constraint $\alpha$; (2) with a sample of clusters drawn from an all-sky hydrodynamical simulation, we will check the effect of cluster asymmetries and dynamical state on the final results. The final maps were constructed using the HealPix package (Gorski et al. 2005) with resolution $N_{\text {side }}=1024$.

\subsection{Y-map from X-Ray-selected Clusters}

Our cluster sample contains 623 clusters outside WMAP Kp0 mask. It was created by combining the ROSAT-ESO Flux Limited X-ray catalog (Böhringer et al. 2004) in the southern hemisphere, the extended Brightest Cluster Sample (Ebeling et al. 1998, 2000) in the north, and the Clusters in the Zone of Avoidance (Ebeling et al. 2002; Kocevski et al. 2007) sample along the Galactic plane. All three surveys are X-ray-selected and X-ray flux limited. A detailed description of the creation of the merged catalog is given in Kocevski \& Ebeling (2006). The position, flux, X-ray luminosity, and angular extent of the region containing the measured X-ray flux were determined directly from the ROSAT All Sky Survey (RASS). All clusters have spectroscopically measured redshifts. The X-ray temperature was derived from the $L_{X}-T_{X}$ relation of White et al. (1997). The central electron densities and core radii were derived by fitting to the RASS data a spherically symmetric isothermal $\beta$-model (Cavaliere \& Fusco-Femiano 1976) convolved with the RASS point-spread function. The $\beta$ was fixed at the canonical value of $2 / 3$ to reduce the dependence of the $\beta$-model parameters with the choice of radius over which the model is fit. These data are sufficient to compute the Comptonization parameter at the center of the cluster.

Atrio-Barandela et al. (2008) compared the TSZ predicted from the X-ray data with the signal present in WMAP three year data and found it to be in good agreement within the X-ray emitting region, where the $\beta$-model is a good description of the electron distribution. In the cluster outskirts, the predicted TSZ signal was systematically higher than the measured value. The latter was consistent with the Komatsu \& Seljak (2002) profile, where baryons are in hydrostatic equilibrium within a dark matter halo well described by a Navarro-Frenk-White profile (hereafter NFW, Navarro et al. 1997), as expected in the concordance $\Lambda$ CDM model. More recently, Nagai et al. (2007) proposed a scaled three-dimensional electron pressure profile $p(x)=P_{e}(r) / P_{500}$ based on a generalized NFW profile:

$$
p(x)=\frac{P_{0}}{\left(c_{500} x\right)^{\gamma}\left[1+\left(c_{500} x\right)^{\alpha}\right]^{(\beta-\gamma) / \alpha}},
$$

where $(\gamma, \alpha, \beta)$ are the central, intermediate, and outer slopes, $c_{500}$ characterizes the gas concentration, and $x=r / R_{500}$ is the distance from the center of the cluster in units of the radius at which the average density of the cluster is 500 times the critical density. Arnaud et al. (2010) derived an average cluster pressure profile from observations of a sample of 33 local $(z<0.2)$ clusters, scaled by mass and redshift with

$$
\begin{aligned}
& {\left[P_{0}, c_{500}, \gamma, \alpha, \beta\right]} \\
& \quad=\left[8.403 h_{70}^{-3 / 2}, 1.177,0.3081,1.0510,5.4905\right]
\end{aligned}
$$

Later, Plagge et al. (2010) showed these parameters to be consistent with the SZ measurements of 15 massive X-ray clusters observed with the South Pole Telescope (Plagge et al. 2010). We determine the scale $R_{500}$ using the Böhringer et al. (2007) relation:

$$
\begin{aligned}
R_{500}= & \frac{(0.753 \pm 0.063) h^{-1} \mathrm{Mpc}}{h(z)} \\
& \times\left(\frac{L_{X}}{10^{44} h^{-2} \mathrm{erg} \mathrm{s}^{-1}}\right)^{0.228 \pm 0.015} .
\end{aligned}
$$

To test the effect of the cluster profile on the final results, we construct $y$-maps from the X-ray cluster catalog (1) using the universal pressure profile of Equation (3) with the parameters given in Equation (4) and (2) using the isothermal $\beta=$ $2 / 3$ model. The Comptonization parameter is computed by integrating the electron pressure profile along the line of sight. Clusters are assumed to be spherically symmetric and extending up to $R_{200}$, the scale where the cluster overdensity reaches 200 times the critical density. To determine the effect of the cluster profile, the central value of $y_{c}$ is assumed to be the same for both the $\beta$-model and the universal pressure profile. Finally, the cluster templates are convolved with the corresponding antenna beams (see Table 1). In Figure 3(a) we 

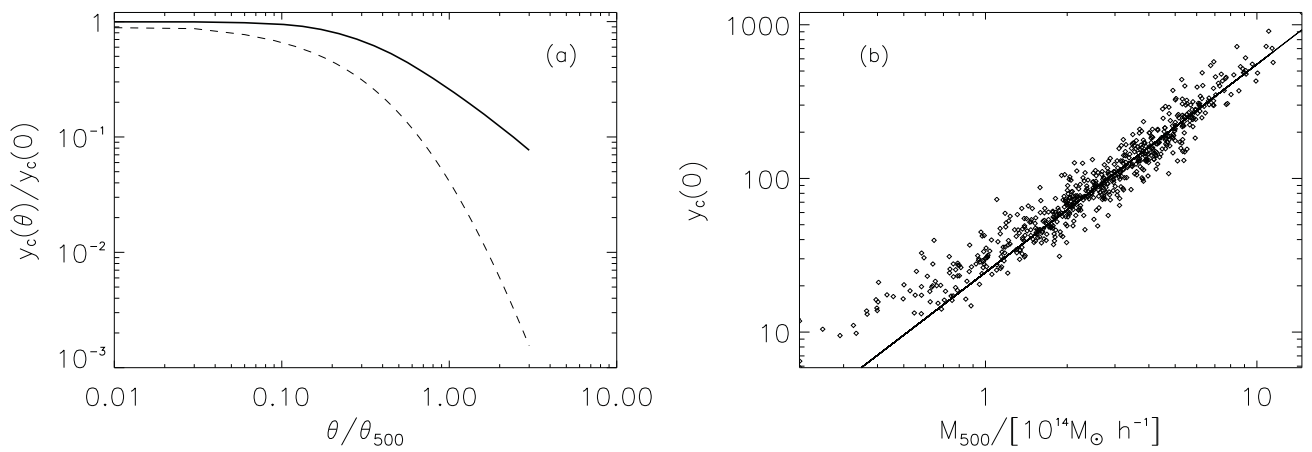

Figure 3. (a) Pressure profile integrated along the line of sight of a cluster with $z=0.094$, of $M_{500}=2.4 \times 10^{14} h^{-1} M_{\odot}$ and $R_{500}=750 h^{-1} \mathrm{Kpc}$ convolved with the antenna of the $44 \mathrm{GHz}$ channel. The solid line corresponds to the $\beta=2 / 3$ model and the dashed line to the universal pressure profile with the parameters of Equation (4). Angles are expressed in units of $\theta_{500}$, the angle subtended by the radius $R_{500}$ at the position of the cluster. (b) Central value of the Comptonization parameter for the clusters of our proprietary sample, derived using the measured X-ray information. The solid line corresponds to the linear regression fit to the data.

show the pressure profile integrated along of line of sight for the $\beta=2 / 3$ (solid line) and universal pressure (dashed line) profiles convolved with the antenna of the $44 \mathrm{GHz}$ map. Since the latter profile diverges at $x=0$, we restrict the integration of Equation (3) to $\theta \geqslant 10^{-2} \theta_{500}$. The cluster is located at $z=0.094$ of $M_{500}=2.4 \times 10^{14} h^{-1} M_{\odot}$ and $R_{500}=746 h^{-1} \mathrm{Kpc}$. For illustration, in Figure 3(b) we plot the value of Comptonization parameter $y_{c}$ at the center of all the clusters in our proprietary cluster catalog, derived using the measured X-ray parameters, as a function of cluster mass. The solid line represents the linear regression fit to the data. The central Comptonization parameter scales as $y_{c}=24.5\left(M_{500} / 10^{14} h^{-1} M_{\odot}\right)^{1.35}$.

\subsection{Y-map from Simulated Clusters}

As an alternative, we also use the low-redshift all-sky maps and the associated galaxy cluster catalogs of the hydrodynamic diffuse and kinetic SZ simulations included in the pre-launch Planck Sky Model. The simulations are fully described in Delabrouille et al. (2012). The catalogs contain cluster positions, mass, and radius for an overdensity contrast of 200 times the critical density. The maps contain the integrated SZ signal up to $z \simeq 0.25$, computed from a combination of full hydrodynamic simulations using the box stacking method described in A. Valente et al. (2012, in preparation). According to this method, the universe around the observer is generated in concentric layers, each with a comoving thickness of $100 h^{-1} \mathrm{Mpc}$, using the outputs of hydrodynamic simulations with periodic boundary conditions. The light-cone integrations of the TSZ and KSZ signals are carried out using the formulae in da Silva et al. (2000, 2001). A total of seven layers were constructed, up to $z=0.25$. The innermost layer includes the local constrained simulation of Dolag et al. (2005), whereas all the other layers were produced from gas snapshots of the $\Lambda$ CDM simulation in De Boni et al. (2011). Both these simulations include explicit treatment for gas cooling, heating by UV, star formation, and feedback processes.

The $y$-map constructed from the X-ray-selected clusters assumes clusters to be spherically symmetric and relaxed while the TSZ and KSZ templates constructed from the hydro-simulation contain clusters with different dynamical state (relaxed, merging systems, etc.), shape, and ellipticity. Also, since the latter are constructed integrating the signal along the line of sight, the projection effects due to low mass clusters and groups are included. Therefore, these templates are very well suited to study the effect of all these systematics and of the KSZ component in the determination of $\alpha$. For a more realistic comparison, we se- lect 623 clusters from the simulation according to the measured selection function of the X-ray cluster sample. In Figure 4(a) we plot the mass and in Figure 4(b) the redshift distribution of all clusters in our simulation (solid line) that fulfill the selection criteria. The dashed line shows the same distributions of the $\mathrm{X}$-ray clusters. For a better comparison, the histogram of the largest amplitude was normalized to unity. The main difference between the two samples is that there are 22 clusters in our proprietary cluster catalog that have redshifts larger than $z=0.25$, the redshift of the last layer constructed from the simulation.

\subsection{Final Maps}

The $y$-maps described above are multiplied by $G(v, \alpha=0)$ to generate TSZ templates and convolved with the antenna beam. Since the cluster catalog contains X-ray temperatures for all clusters, in the template generated from the sample of real clusters we included relativistic corrections using the analytic formulae derived by Nozawa et al. (2000) that are accurate for clusters with temperature $T_{X} \leqslant 25 \mathrm{KeV}$ at all the frequencies measured by Planck. A KSZ template was added to the hydrodynamical but not to the X-ray-selected cluster template since their peculiar velocity are not available. Noise maps were constructed assuming the Blue Book noise levels of Table 1. We model the noise as homogeneous and uncorrelated white noise since at the frequencies $44-353 \mathrm{GHz}$ the $1 / f$ is both small and does not affect the angular scales subtended by clusters, $\ell \geqslant 500$.

To construct the final maps for the two types of data (A) and (B) we add different contributions. To make the simulations as realistic as possible we need to consider $\mathrm{CMB}$ and foreground residuals, noise inhomogeneities, and deviations from white noise behavior. For instance, when averaged over the cluster extent, CMB residuals do not scale down as $N_{\text {pixel }}^{-1 / 2}$ and, together with foreground residuals, they can induce systematic shifts in the Comptonization parameter. The effect will be dependent on both amplitude and shape of the residual power spectrum. CMB residuals dominate over the noise up to $\ell \sim 2000$ on the 70-353 GHz channels (see Leach et al. 2008, Figure 6). Therefore, one can expect that these residuals would dominate over noise inhomogeneities or $1 / f$ contributions and so we will not consider the latter in our simulations. With respect to foreground residuals, they change with frequency, have different origin, different amplitude and are irregularly distribute in the sky. Further, only low-resolution $\left(\sim 1^{\circ}\right)$ templates are available for synchrotron and free-free emission (Leach et al. 2008) so the behavior of the foregrounds, not just foreground residuals, on 


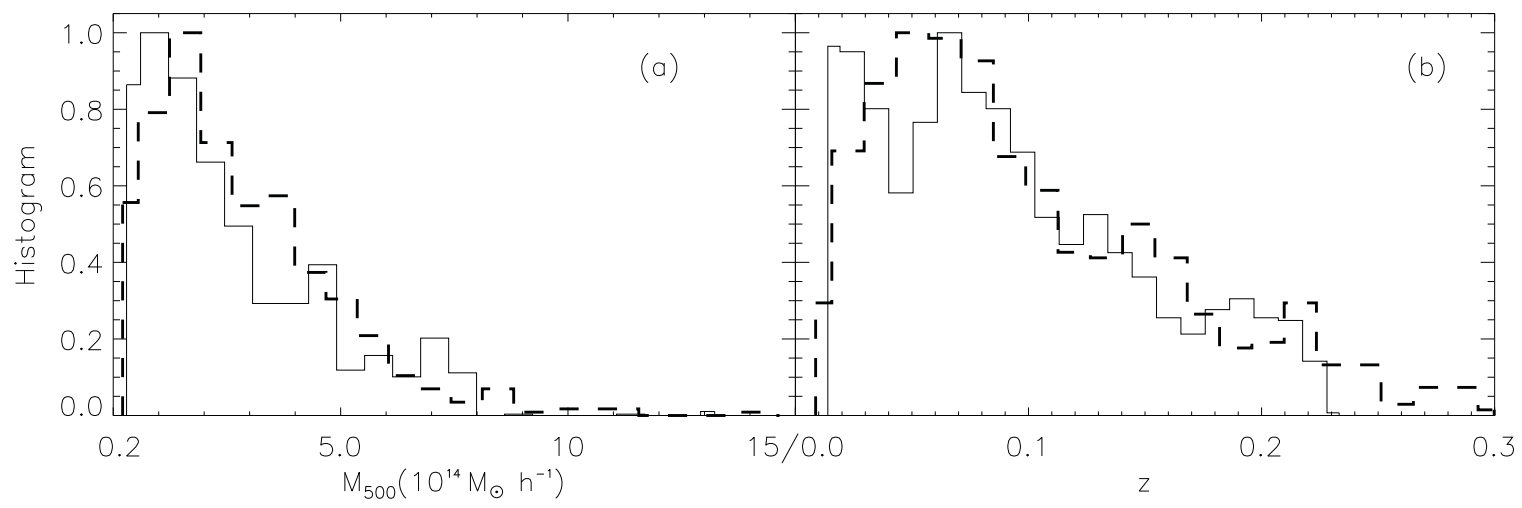

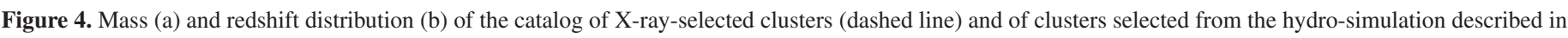
Section 3.2. For easier comparison, the histograms are normalized to unity at the maximum.

scales $<1^{\circ}$ relevant for the study of clusters, cannot be modeled accurately. However, Galactic emissions are not associated with clusters, their effect on the measured value of $\alpha$ can be analyzed by studying different cluster subsamples in different regions of the sky. Other foregrounds associated with clusters could have a more damaging effect. Diffuse and point-like radio emission can be found in cluster cores. Their physical origins are very different though and both are rare, in particular among the very $\mathrm{X}$-ray luminous clusters that dominate the signal discussed by us here (Brunetti et al. 2007). Specifically, radio halos are found only in merging clusters (Cassano et al. 2010). Radio emission from individual cluster galaxies is usually limited to the brightest cluster galaxy and, opposite to the case of diffuse emission, observed almost exclusively in relaxed systems. Like for other foregrounds, the contribution of both effects could be estimated by analyzing different cluster subsamples, binning by redshift or by galactic latitude. In any case, all these effects can only be accurately modeled once the data become available.

In comparison, $\mathrm{CMB}$ residuals represent an extreme case of foreground: they are the same at all frequencies, except by the change in amplitude due to the dilution by the beam, and they are distributed everywhere in the sky. In this context, CMB residuals are the most difficult to separate so they will be the only ones included in our simulations. Since different component separation methods will leave different residuals, we adopted the filter described in Kashlinsky et al. (2009) to create our CMB residual template. This filter is designed to remove the primary $\mathrm{CMB}$ fluctuations from the concordance $\Lambda \mathrm{CDM}$ model by minimizing the mean squared deviation of the $\mathrm{CMB}$ measurements from noise $\left\langle\left(\delta_{\mathrm{CMB}}-\text { noise }\right)^{2}\right\rangle$, and it generates $\mathrm{CMB}$ residuals that are homogeneously distributed in the sky. In this respect we are being conservative; the true residuals are likely to be smaller away from the galactic plane (see Planck HFI Core Team 2011b, Figure 39), fact that could permit to estimate the effect of the residuals by analyzing cluster subsamples selected according to galactic latitude.

To summarize, we constructed two sets of maps, according to the specific data sets: (A) CMB subtracted using a template, (B) CMB subtracted exactly using the $217 \mathrm{GHz}$ channel. In simulation (A) six maps are constructed, adding to the TSZ template CMB residuals and white noise. The noise is generated according to the parameters given in Table 1 . In all the simulations the residual CMB map is kept fixed and only the noise varies. In this form we can quantify the bias introduced by the residuals. We took residual maps with amplitude $\left\langle\left(\Delta T_{\mathrm{CMB}, \mathrm{res}}\right)^{2}\right\rangle^{1 / 2}=$ $1,10 \mu \mathrm{K}$. For the $y$-map constructed from real clusters, only the
TSZ signal was included. The temperature anisotropy at each pixel is $\Delta T_{A}(v)=y_{c} G(v, 0)+\Delta T_{\mathrm{TSZ}} \pm \sigma_{\text {noise }, v}^{A} \pm \Delta T_{\mathrm{CMBres}}$. For the $y$-map derived from simulations, we also included a KSZ component. In simulation (B) six maps are constructed adding a cosmological CMB signal and noise to the $y$-map. The $217 \mathrm{GHz}$ map is used to subtract the cosmological CMB and KSZ signals exactly. Therefore, only five different maps are available for the analysis. We checked the final maps had a power spectrum that was a pure white noise, with a slightly larger variance $\sigma_{\text {noise, } v}^{B}$, sum of the original map plus the noise of the degraded $217 \mathrm{GHz}$ map. At each pixel, the temperature anisotropy is $\Delta T_{B}(\nu)=y_{c} G_{[-217 \mathrm{GHz}]}(\nu, 0) \pm \sigma_{\text {noise }, \nu}^{B}$.

\section{DATA PROCESSING}

In both simulations (A) and (B), we construct estimators using both the ratio and the fit methods. To simplify the notation, let the index $I=(A, B)$ denote the type of simulation and let us redefine $G_{A}=G(\nu, \alpha), R_{A}=R\left(v_{1}, v_{2}, \alpha\right), G_{B}=$ $G_{[-217 \mathrm{GHz}]}(\nu, \alpha)$, and $R_{B}=R_{[-217 \mathrm{GHz}]}\left(v_{1}, v_{2}, \alpha\right)$. At each cluster location, projection effects can yield contributions from different redshifts altering the frequency dependence. To reduce the effect, we average over the cluster extent.

\subsection{Ratio Method}

In this method we take the ratio of the temperature anisotropy at two different frequencies. Maps are brought to a common resolution before taking the ratio. Since the ratio of two Gaussian random variables is not a Gaussian, we estimate $\alpha$ using the approach introduced by Luzzi et al. (2009). If the average temperature anisotropy at two different frequencies $i=1,2$ at one cluster location $j$ are denoted by $\delta_{j}\left(v_{i}\right)=\left\langle\Delta T_{j, I}\left(v_{i}\right)\right\rangle$, and they are measured with Gaussian errors $\sigma_{i}$ then for each cluster $j$, the probability $P_{j}\left(R_{I}\right)$ that the ratio $R_{I}\left(v_{1}, v_{2}\right)=\delta_{1} / \delta_{2}$ of two temperature anisotropies is in the range $(r, r+d r)$ is given by

$$
\begin{aligned}
& P_{j}\left(R_{I}\left(v_{1}, v_{2}, \alpha\right)\right)=\frac{1}{2 \pi \sigma_{1} \sigma_{2}} \\
& \quad \times \int_{-\infty}^{\infty} x \exp \left(-\left[\frac{\left(x-\delta_{1}\right)^{2}}{2 \sigma_{1}^{2}}+\frac{\left(x R_{I}\left(v_{1}, v_{2}, \alpha\right)-\delta_{2}\right)^{2}}{2 \sigma_{2}^{2}}\right]\right) d x .
\end{aligned}
$$

The most likely value of $\alpha$ is then given by the maximum of the likelihood function:

$$
\log \mathcal{L}=\sum_{v_{1}, \nu_{2}} \sum_{j=1}^{N_{c l}} \log \left[P_{j}\left(v_{1}, v_{2}, \alpha\right)\right] .
$$



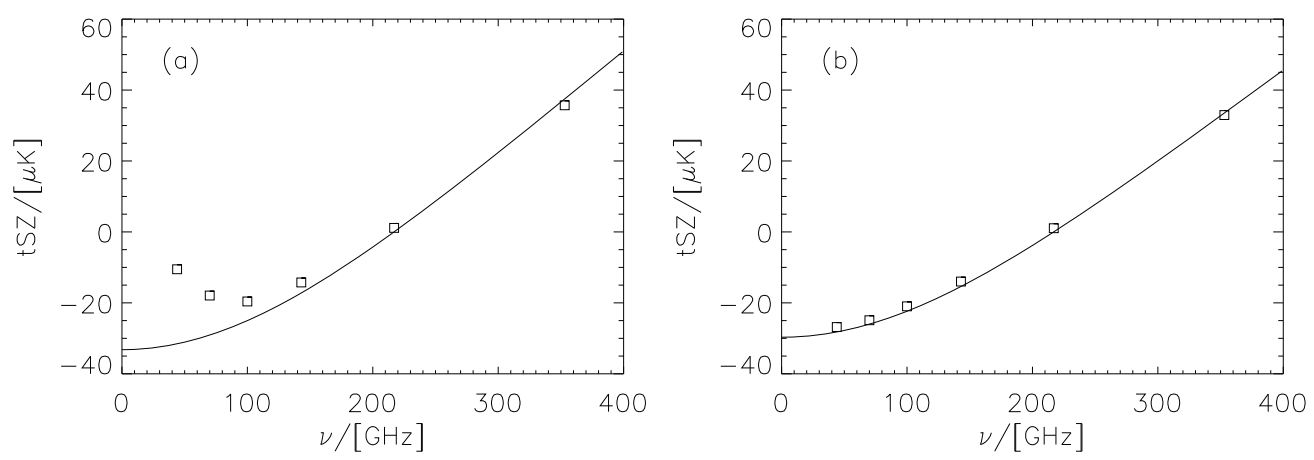

Figure 5. Effect of the beam dilution on the spectral dependence of the TSZ effect. Open squares represent the amplitude of the cluster TSZ effect at different frequencies, averaged over a disk of extent $2 \theta_{500}$. The solid line represents the TSZ scaling $\left.G_{(} \nu, 0\right)$. Panel (a) corresponds to a cluster of mass $M_{500}=3.64 \times 10^{14} h^{-1} M_{\odot}$ at redshift $z=0.218$, that subtends an angle $\theta_{500}=9$ '.4. Panel (b) corresponds to $M_{500}=7.7 \times 10^{14} h^{-1} M_{\odot}$ located at redshift $z=0.058$, subtending an angle $\theta_{500}=42^{\prime}$.
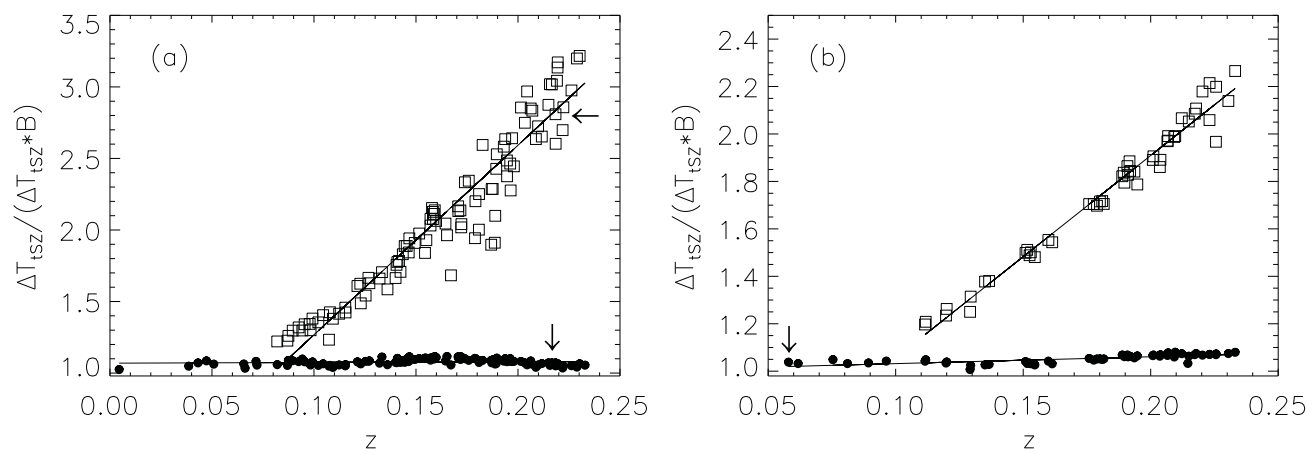

Figure 6. (a) Deconvolution factor for clusters in the mass range $M_{500}=5-6 \times 10^{14} h^{-1} M_{\odot}$ and (b) for clusters with $M_{500} \geqslant 1 \times 10^{15} h^{-1} M_{\odot}$. Solid black circles represent the deconvolution factor for the $353 \mathrm{GHz}$ channel and open squares for the $44 \mathrm{GHz}$ channel. All clusters are resolved at $353 \mathrm{GHz}$ but, for simplicity, at $44 \mathrm{GHz}$ only the fraction of unresolved clusters is shown. Arrows indicate the deconvolution factor of the clusters of Figure 5.

Luzzi et al. (2009) demonstrate that the ratio of the two distributions is biased; the ratio is dominated by the error on the denominator. For this reason, we use the denominator to be the measurement with the smallest fractional errors in order to minimize the bias, i.e., the 100 and $143 \mathrm{GHz}$ channels. In total we have nine different ratios.

\subsection{Frequency Fit Method}

Alternatively, we can fit the TSZ signal of each cluster to the spectral dependence of Figures 1(b) and 2(b). In this case, errors are Gaussian distributed and the likelihood function is given by

$$
-2 \log \mathcal{L}=\sum_{\nu} \sum_{j=1}^{N_{c l}}\left[\frac{\left\langle\Delta T_{I}(v)\right\rangle-\bar{y}_{c} G_{I}(v, \alpha)}{\sigma_{\text {noise }, v, j}^{I}}\right]^{2},
$$

where $\sigma_{\text {noise, }, i}^{I}$ is the error at each cluster location that includes noise and $\mathrm{CMB}$ residuals (simulation (A)) or just noise (simulation (B)).

In this method, since we do not measure $G(v, \alpha)$ directly but $\Delta T(\hat{n})=T_{0} y_{c} G(\nu, \alpha)$, we need an independent determination of $y_{c}$ using X-ray data, introducing another complication: different frequencies have different resolutions and cluster anisotropies are diluted differently by the antenna beam. The amplitude of the effect depends on the cluster profile and angular extent but does not depend on the scaling of the TSZ signal with redshift, $G(v, \alpha)$. To show this effect, in Figure 5 we represent the effect for two different clusters. Figure 5(a) corresponds to a cluster at redshift $z=0.218$, with mass $M_{500}=$ $3.64 \times 10^{14} h^{-1} M_{\odot}$ and size 9'.4 while in Figure 5(b) the cluster is located at $z=0.058$ with mass $M_{500}=7.7 \times 10^{14} h^{-1} M_{\odot}$ and size $42^{\prime}$. The dilution effect is most noticeable at $44 \mathrm{GHz}$ since this channel has the smallest resolution.

For clusters drawn from a simulation, its size, ellipticity, and profile are well known. For such clusters, the deconvolution factor $F$ can be determined exactly by comparing the average Comptonization parameter before, $\left\langle y_{c}\right\rangle$, and after, $\left\langle y_{c} * B(v)\right\rangle$, convolving with the antenna beam $B(v): F=\left\langle y_{c}\right\rangle /\left\langle y_{c} * B(v)\right\rangle$. This factor would be different for resolved and unresolved clusters and would depend on the cluster profile and redshift. For illustration, in Figure 6(a) we represent $F$ for a subset of 110 clusters in the mass range $M_{500}=5-6 \times 10^{14} h^{-1} M_{\odot}$. In Figure $6(\mathrm{~b})$, we plot the deconvolution factors for all clusters in our simulation with masses $M_{500} \geqslant 10^{15} h^{-1} M_{\odot}$. Solid black circles correspond to the $353 \mathrm{GHz}$ frequency and open squares to $44 \mathrm{GHz}$. All clusters are resolved at $353 \mathrm{GHz}$. At $44 \mathrm{GHz}$ clusters with redshift $z \geqslant 0.08$ are unresolved. For clarity, the resolved clusters are not shown. The solid straight lines correspond to the linear regression fit, $F_{\text {lin }}$, to the deconvolution factor, $F$, different for each cluster mass range and channel. Arrows indicate the deconvolution factor of the clusters plotted in Figures 5(a) and (b).

If $\Delta F$ is the rms dispersion of the true deconvolution values $F$ around $F_{\text {lin }}$ for each mass bin and antenna, then $F=$ $F_{\text {lin }} \pm \Delta F$. We used the $y$-map computed with clusters drawn from a numerical simulation to compute the deconvolution factors $F_{\text {lin }}$ and its uncertainty $\Delta F$ in three mass bins of equal number of clusters: $M_{500} \leqslant 2 \times 10^{14} h^{-1} M_{\odot}, M_{500}=$ $2-3.6 \times 10^{14} h^{-1} M_{\odot}$, and $M_{500} \geqslant \times 3.6 \times 10^{14} h^{-1} M_{\odot}$. These deconvolution factors were later used to deconvolve the effect of the beam on the sample of X-ray-selected clusters. If for a real cluster we use $F_{\text {lin }}$ instead of the (unknown) true factor $F$, the 

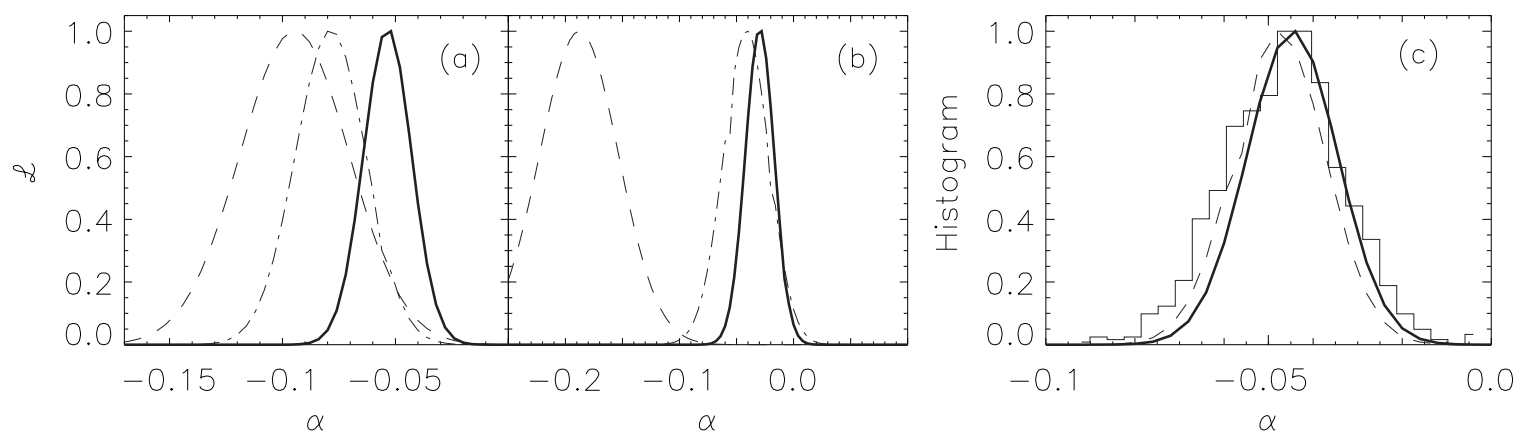

Figure 7. Ratio method with simulation (A). Likelihood function for subsamples of 623 clusters distributed (a) in three redshift bins $z=([<0.11]$, [0.11-0.17], [>0.17]) of equal number of clusters and (b) in three mass bins $M_{500}=([\leqslant 1.9],[0.19-0.37],[\geqslant 3.7]) \times 10^{14} h^{-1} M_{\odot}$ also with the same number of clusters. In both plots, dashed, dot-dashed, and solid lines correspond to low, intermediate, and high redshift/mass bins. (c) Dashed line: full likelihood of the single simulation (a) and (b), the histograms of the value of $\alpha$ derived from 1000 simulations, arbitrarily normalized to unity and the thick solid line is a Gaussian fit to the histogram.

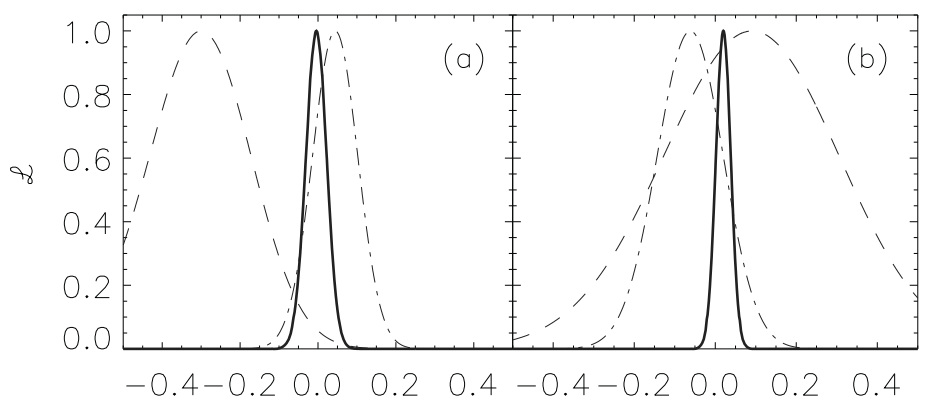

$\alpha$

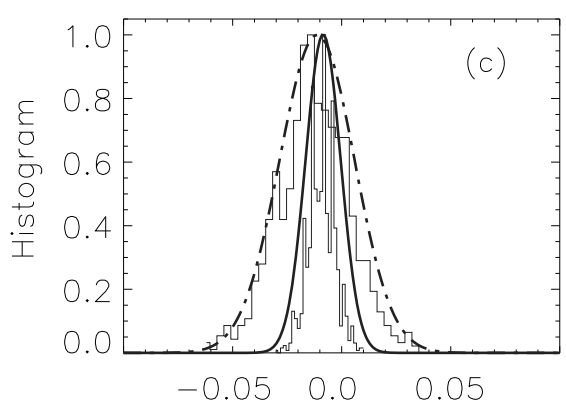

$\alpha$

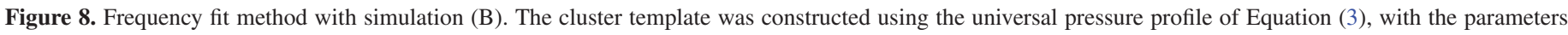

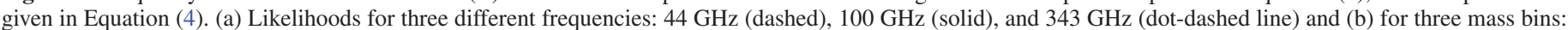

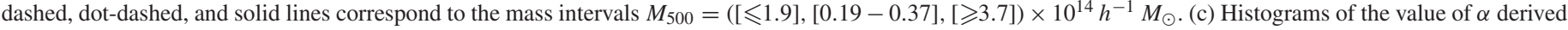

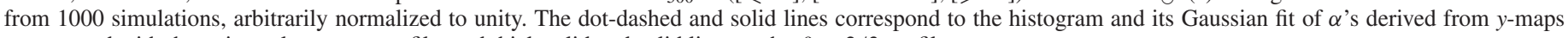
constructed with the universal pressure profile, and thick solid and solid lines to the $\beta=2 / 3$ profile.

deconvolved signal $\left(\Delta T_{\mathrm{TSZ}} * B\right) F_{\text {lin }}$ would differ from the true signal $\Delta T_{\mathrm{TSZ}}$ by an amount $\left(\Delta T_{\mathrm{TSZ}} * B\right) \Delta F$. This uncertainty is uncorrelated with the instrumental noise at the cluster location and can be included in the likelihood analysis of Equation (8) by adding it in quadrature: $\sigma_{\text {tot }, i}^{2}=\sigma_{\text {noise }, i}^{2}+\left[\left(\Delta T_{\mathrm{TSZ}} * B\right) \Delta F\right]^{2}$. On the negative side, the deconvolution coefficient does not scale linearly with redshift, and $F_{\text {lin }}$ underestimates the true deconvolution factor $F$ especially at high redshifts, potentially biasing our estimation of $\alpha$. The effect would be more noticeable at $44 \mathrm{GHz}$, since the linear factor would underestimate the correction at small redshifts and overestimate it at higher redshifts (see the discussion of Figure 8(a) below).

\section{RESULTS AND DISCUSSION}

We first tested the ratio and fit methods using a template constructed from simulations, as described in Section 3.2. The template contained a subset of 623 clusters distributed in mass and redshift according to the cluster catalog selection function (see Figure 4) Second, we repeated the analysis with the template of X-ray-selected clusters. For the sample of real clusters the template was constructed using the universal pressure profile of Equation (3), with the parameters given in Equation (4) but also with the $\beta$-model profile, with $\beta=2 / 3$. The first and most important conclusion is that we found no significant differences from the results obtained using the simulated cluster template or the universal pressure profile template, implying that the effect of KSZ, cluster dynamical state, and deviations from spherical symmetry are averaged out over such a large sample of clusters. These effects were important when analyzing observations of just a few clusters (Battistelli et al. 2002; Luzzi et al. 2009). For real clusters, the $\beta$-model profile provides better constraints on $\alpha$ than the universal pressure profile. This is consequence of the TSZ profile falling less steeply for the former than for the latter (Atrio-Barandela et al. 2008). With respect to the method of analysis, the ratio method performs differently if there are CMB residuals, but the differences are small for the fit method. For the ratio method, we present the results with $\mathrm{CMB}$ residuals of amplitude $1 \mu \mathrm{K}$ (simulation type (A), Figure 7); for the fit method, CMB is removed using the $217 \mathrm{GHz}$ map (simulation type (B), Figure 8) and we discuss all other cases.

To test the importance of the different contributions, we define three mass bins $M_{500}=([<0.192],[0.192-0.365]$, [>0.36]) $\times 10^{15} M_{\odot} h^{-1}$ of equal number $(\sim 208)$ of clusters, and three redshift bins $z=([<0.11],[0.11-0.17],[>0.17])$ with mean redshift $\langle z\rangle=(0.08,0.14,0.20)$, also with the same number of clusters. We computed the likelihood (Equations (7) and (8)) for the different mass and redshift bins and different frequencies to determine the data subset with the largest statistical power. To compute likelihoods, we subdivide the interval $\alpha=[-1,1]$ in 2001 steps. We perform 1000 Monte Carlo simulations for each cluster template, simulation type, and method.

\subsection{Ratio Method}

In Figure 7 we present the likelihood function of a single simulation randomly selected of our ensemble of 1000 simulations. In Figure 7(a) we represent the likelihood for clusters within the three redshift bins given above, marginalized over cluster mass, and nine frequency ratios. Dashed, dot-dashed, and solid lines correspond to the lower, intermediate, and high redshift bins. 
For the latter, $\alpha=-0.052 \pm 0.011$. In Figure 7(b) we present the results binning clusters according to mass. Dashed, dotdashed, and solid correspond to lower, intermediate, and high mass bins. As expected, the most massive clusters dominate the likelihood. The value $\alpha$ in this single simulation measured from the bin containing the most massive clusters is $\alpha=-0.028 \pm 0.013$. The full likelihood, including all redshifts and all masses, is represented in Figure 7(c) (dashed line). We also plot the distribution of the values of $\alpha$ measured in the thousand simulations (histogram). The thick solid line is a Gaussian fit to the histogram. For the single simulation, we obtain $\alpha=-0.048 \pm 0.010$ while the average over simulations is $\bar{\alpha}=-0.045, \sigma_{\bar{\alpha}}=0.010$. By keeping fixed the template of $\mathrm{CMB}$ residuals we verified that the effect of CMB residuals is to bias our estimate of $\alpha$ since the rms dispersion in $\alpha$ equals the error in $\alpha$ in one single realization. In this particular case, $\alpha$ is biased to negative values, but for different realizations of the residuals, the bias could be positive with equal probability. If the $\mathrm{CMB}$ residual is as large as $\Delta T_{\mathrm{CMBres}}=10 \mu \mathrm{K}$, then the bias rises to $\bar{\alpha}=0.6$. In the ideal case that the CMB template removes the cosmological signal exactly and no $\mathrm{CMB}$ residuals are present in the data, the result would be $\bar{\alpha}=-0.003 \pm 0.011$; the error bar is similar but the bias becomes negligible.

The ratio method is biased both by the error in the denominator (Luzzi et al. 2009) and also by CMB and foreground residuals present in the data. For this method to provide an unbiased estimate of $\alpha$, the residual contribution due to CMB and astrophysical foregrounds must be smaller than $\sim 1 \mu \mathrm{K}$. The bias can be estimated by analyzing clusters selected by galactic latitude, since, as indicated, $\mathrm{CMB}$ and foreground residuals are largest close to the galactic plane (see Planck HFI Core Team 2011b). Also, we checked that ratios using lower resolution maps have less statistical power to constrain $\alpha$. In retrospect, this justifies neglecting the 30,545 , and $857 \mathrm{GHz}$ channels, with large noise levels or $(1 / f)$ contributions, that would complicate the analysis without adding more information.

\subsection{Fit Method}

The results of the fit method using the $217 \mathrm{GHz}$ map to remove the intrinsic CMB are presented in Figure 8. In panel (a) we plot the likelihood function of a single simulation for three different frequencies: $44 \mathrm{GHz}$ (dashed), $100 \mathrm{GHz}$ (solid), and $343 \mathrm{GHz}$ (dot-dashed line). The figure shows that the $100 \mathrm{GHz}$ channel is the most restrictive and the $44 \mathrm{GHz}$ channel is the less restrictive of the three. The latter is also biased since the deconvolution factor is the largest of all the Planck channels (see Figure 5) and is not as well represented by a linear approximation with a small correction, especially at low masses (see Figure 6(a)). The biased introduced does not alter significatively the final results since the final likelihood is also dominated by the channels that have the highest resolution and lowest noise, in this case the 100 and $143 \mathrm{GHz}$ channels. In Figure 8(b) we represent the likelihood for the three mass bins given above and marginalized over frequencies; dashed, dot-dashed, and solid lines correspond to the low, intermediate, and high mass intervals. The signal is dominated by the most massive clusters that, on a flux-limited sample, are, on average, at high redshift than the lower and intermediate mass samples. For this particular realization, the value estimated from the subsample of most massive clusters is $\alpha=0.020 \pm 0.018$, adiabatic evolution being only marginally outside the $1 \sigma$ level.

In Figure 8(c) we represent the histograms of 1000 simulations together with their linear fits for cluster templates con- structed using the universal pressure profile (dot-dashed line) and the $\beta=2 / 3$ (solid line). The mean and rms dispersion of the estimated values are $\langle\alpha\rangle=-0.011 \pm 0.018$ for the universal profile and $\langle\alpha\rangle=0.009 \pm 0.008$ for the $\beta$-model profile. When all cluster properties are identical, the TSZ integrated over the cluster extent will be larger for the $\beta$-model than for the universal profile (see Figure 3(a)) so it must constrain $\alpha$ better, as shown. We also carried out 1000 simulations using method (A) with the hydrodynamical template, which contains the KSZ component and CMB residuals of $1 \mu \mathrm{K}$ amplitude. The result was $\langle\alpha\rangle=-0.008 \pm 0.015$, identical to the result with method (B) above. Therefore, in the fit method is not so important to have maps free of residuals as, for example, in the ratio method. Using the $217 \mathrm{GHz}$ map to remove the intrinsic CMB signal alters the TSZ frequency dependence, but the TSZ signal is still strongly dependent with redshift (see Figures 1(b) and 2(b)) what is not the case in the ratio method (compare Figures 1(a) and 2(a)) and an extra error of $\sim 1 \mu \mathrm{K}$, even if correlated across frequencies, is not so significant.

Let us remark that the rms dispersion of $\alpha$ on 1000 simulations, $\sigma_{\alpha}$, is very similar to the error on $\alpha$ in one single realization, both in the ratio and in the fit method, indicating that our pipelines are efficient. The results obtained using $y$-maps constructed with clusters drawn from a hydrodynamical simulation or from a catalog of X-ray-selected clusters show no significant differences. In the hydro-simulation, the $y$-map integrates the SZ signal up to $z \simeq 0.25$ and contains all the projection effects up to that redshift, not included in the X-ray-selected cluster template; then, we can conclude that projection effects play no significant role. This can be understood in the light of the results presented in Figure 8(b); the full likelihood is dominated by the most massive clusters for which projection effects are not significant (see A. Valente et al. 2012, in preparation for a discussion on this point).

\section{CONCLUSIONS}

Planck offers an excellent opportunity to constrain the evolution history of the CMB blackbody temperature with better precision than quasar excitation lines using currently available $\mathrm{X}$-ray cluster catalogs. We have found that taking the ratio of temperature anisotropies at different frequencies is biased. For the method to be of use, it requires the level of foreground and $\mathrm{CMB}$ residuals to be smaller than $\sim 1 \mu \mathrm{K}$. Fitting the frequency dependence provides an alternative estimator, with different systematics but more difficult to implement since it requires both an independent determination of the Comptonization parameter and to deconvolve the antenna beam. The latter cannot be done exactly if the cluster pressure profile is not known precisely. We have shown that deconvolution using linear fits introduces an error that can be easily incorporated into the analysis.

We have considered two possible methods to remove foregrounds and the cosmological CMB signal and the KSZ contribution: using a CMB template, as described in Leach et al. (2008) or using the $217 \mathrm{GHz}$ map. We have carried out simulations of both methods to investigate the differences on the final results. We have shown that the ratio method performs rather well if the cosmological CMB signal is removed exactly, but the method is biased if a level of residuals of amplitude $\sim 1 \mu \mathrm{K}$ or larger are present. The fit method performs equally well in both data sets, giving results that are only marginally biased. However, since both methods have different systematics, they will be complementary to each other. In particular, massive clusters and the highest resolution/lowest noise channels have the 
largest statistical power to constrain $\alpha$ and subsets at different redshifts, mass ranges, or galactic latitudes could be used to reduce the bias. We have used a proprietary cluster catalog that contains spectroscopic redshifts and all the required X-ray information to estimate the accuracy that would be achieved with Planck data. We forecast that the final uncertainty will be about 0.011-0.016 a factor of 2-3 better than those obtained from quasar spectra by Noterdaeme et al. (2011), depending on what type of Planck data become publicly available.

Since our catalog is restricted to clusters with $z \leqslant 0.3$, we have not extended our analysis beyond that redshift. Planck has already detected around 200 clusters with an $S / N \geqslant 10$, one at $z \simeq 0.94$ with $M_{500} \simeq 8 \times 10^{14} M_{\odot}$. Adding more clusters with current or future experiments will help to detect possible deviations from adiabatic evolution, especially if clusters are of higher mass and are at a higher redshift like the one recently reported in Planck Collaboration (2011c). Once all PLANCK and South Pole cluster candidates have been observed on the X-ray and their redshift determined, the measurements proposed will provide much stronger constraints on non-adiabatic evolution than those quoted here.

This work was done in the context of the FCT/MICINN cooperation grant "Cosmology and Fundamental Physics with the Sunyaev-Zeldovich Effect" AIC10-D-000443, with additional support from project PTDC/FIS/111725/2009 from FCT, Portugal and FIS2009-07238 and CSD 2007-00050 from the Ministerio de Educación y Ciencia, Spain. The work of C.M. is funded by a Ciência2007 Research Contract, funded by FCT/MCTES (Portugal) and POPH/FSE (EC).

\section{REFERENCES}

Aghanim, N., Hansen, S. H., \& Lagache, G. 2005, A\&A, 439, 901 Arnaud, M., Pratt, G. W., Piffaretti, R., et al. 2010, A\&A, 517, 92

Atrio-Barandela, F., Kashlinsky, A., Kocevski, D., \& Ebeling, H. 2008, ApJ, 675, L57

Avgoustidis, A., Luzzi, G., Martins, C. J. A. P., \& Monteiro, A. M. R. V. L. 2012, J. Cosmol. Astropart. Phys., JCAP02(2012)013

Bahcall, J. N., \& Wolf, R. A. 1968, ApJ, 152, 701

Battistelli, E. S., De Petris, M., Lamagna, L., et al. 2002, ApJ, 580, L101

Böhringer, H., Schuecker, P., Guzzo, L., et al. 2004, A\&A, 425, 367

Böhringer, H., Schuecker, P., Pratt, G. W., et al. 2007, A\&A, 469, 363

Brunetti, G., Venturi, T., Dallacasa, D., et al. 2007, ApJ, 670, L5

Cassano, R., Ettori, S., Giacintucci, S., et al. 2010, ApJ, 721, L82

Cavaliere, A., \& Fusco-Femiano, R. 1976, A\&A, 49, 137

da Silva, A., Barbosa, D., Liddle, A., \& Thomas, P. 2000, MNRAS, 317, 37

da Silva, A., Barbosa, D., Liddle, A., \& Thomas, P. 2001, MNRAS, 326, 155

De Boni, C., Dolag, K., Ettori, S., et al. 2011, MNRAS, 415, 2758
Delabrouille, et al. 2012, The Pre-launch Planck Sky Model: A Model of Sky Emission at Submillimetre to Centimetre Wavelengths, in press (arXiv:1207.3675)

Dolag, K., Hansen, F. K., Roncarelli, M., \& Moscardini, L. 2005, MNRAS, 363,29

Ebeling, H., Edge, A. C., Allen, S. W., et al. 2000, MNRAS, 318, 333

Ebeling, H., Edge, A. C., Böhringer, H., et al. 1998, MNRAS, 301, 881

Ebeling, H., Mullis, C. R., \& Tully, R. B. 2002, ApJ, 580, 774

Etherington, J. M. H. 1933, Phil. Mag., 15, 761

Fabbri, R., Melchiorri, F., \& Natale, V. 1978, Ap\&SS, 59, 223

Finkbeiner, D. P. 2003, ApJS, 146, 407

Finkbeiner, D. P., Davis, M., \& Schlegel, D. J. 1999, ApJ, 524, 867

Gold, B., Bennett, C. L., Hill, R. S., et al. 2009, ApJS, 180, 265

Gorski, K., Hivon, E., Banday, A. J., et al. 2005, ApJ, 622, 759

Horellou, C., Nord, M., Johansson, D., \& Lévy, A. 2005, A\&A, 441, 435

Jetzer, P., Puy, D., Signore, M., \& Tortora, C. 2011, Gen. Rel. Grav., 43, 1083

Jetzer, P., \& Tortora, C. 2012, Phys. Rev., D84, 043517

Kashlinsky, A., Atrio-Barandela, F., Kocevski, D., \& Ebeling, H. 2009, ApJ, 691, 1479

Kocevski, D. D., \& Ebeling, H. 2006, ApJ, 645, 1043

Kocevski, D. D., Ebeling, H., Mullis, C. R., \& Tully, R. B. 2007, ApJ, 662, 224

Komatsu, E., \& Seljak, U. 2002, MNRAS, 336, 1256

Leach, S. M., Cardoso, J.-F., Baccigalupi, C., et al. 2008, A\&A, 491, 597

Lima, J. A. S., Silva, A. I., \& Viegas, S. M. 2000, MNRAS, 312, 747

LoSecco, J. M., Mathews, G. J., \& Wang, Y. 2001, Phys. Rev. D, 64, 123002

Luzzi, G., Shimon, M., Lamagna, L., et al. 2009, ApJ, 705, 1122

Mather, J. C., Fixsen, D. J., Shafer, R. A., Mosier, C., \& Wilkinson, D. T. 1999, ApJ, 512, 511

Matyjasek, J. 1995, Phys. Rev. D, 51, 4154

Mennella, A., Butler, R. C., Curto, A., et al. 2011, A\&A, 536, A3

Mortonson, M., Hu, W., \& Huterer, D. 2009, Phys. Rev. D, 80, 067301

Nagai, D., Kravtsov, A. V., \& Vikhlinin, A. 2007, ApJ, 668, 1

Navarro, J. F., Frenk, C. S., \& White, S. D. M. 1997, ApJ, 490, 493

Noterdaeme, P., Petitjean, P., Srianand, R., Ledoux, C., \& López, S. 2011, A\&A, 526, L7

Nozawa, S., Itoh, N., Kawana, Y., \& Kohyama, Y. 2000, ApJ, 536, 31

Nunes, N. J., Dent, T., Martins, C. J. A. P., \& Robbers, G. 2009, Mem. Soc. Astron. Ital., 80, 785

Overduin, J. M., \& Cooperstock, F. I. 1998, Phys. Rev. D, 58, 043506

Plagge, T., Benson, B. A., Ade, P. A. R., et al. 2010, ApJ, 716, 1118

Planck Collaboration 2011a, A\&A, 536, A1

Planck Collaboration 2011b, A\&A, 536, A8

Planck Collaboration 2011c, A\&A, 536, A26

Planck HFI Core Team 2011a, A\&A, 536, A4

Planck HFI Core Team 2011b, A\&A, 536, A6

Puy, D. 2004, A\&A, 422, 1

Rephaeli, Y. 1980, ApJ, 241, 858

Songaila, A., Cowie, L. L., Hogan, C. J., \& Rugers, M. 1994, Nature, 368, 599

Srianand, R., Petitjean, P., \& Ledoux, C. 2000, Nature, 408, 931

Sunyaev, R. A., \& Zeldovich, Y. B. 1972, Comments Astrophys. Space Phys., 4, 173

Sunyaev, R. A., \& Zeldovich, I. B. 1980, MNRAS, 190, 413

White, D. A., Jones, C., \& Forman, W. 1997, MNRAS, 292, 419

Zacchei, A., Maino, D., Baccigalupi, C., et al. 2011, A\&A, 536, A5

Zemcov, M., Aguirre, J., Bock, J., et al. 2012, ApJ, 749, 114 